\title{
Florística e germinação ex situ do banco de sementes do solo em diferentes níveis de
}

\section{luminosidade}

\author{
Floristics and ex situ germination of the soil seed bank under different light levels \\ Florística y germinación ex situ del banco de semillas del suelo a diferentes niveles de luz
}

Recebido: 27/12/2020 | Revisado: 02/01/2021 | Aceito: 08/01/2021 | Publicado: 09/01/2021

Iracema Maria Cordeiro

ORCID: https://orcid.org/0000-0002-9181-264X

Universidade Federal Rural da Amazônia, Brasil

E-mail: iracema3c@gmail.com

Osmar Alves Lameira

ORCID: https://orcid.org/0000-0001-8370-8562

Embrapa Amazônia Oriental, Brasil

E-mail: osmar.lameira@embrapa.br

Raphael Prado Neves

ORCID: https://orcid.org/0000-0002-0997-4851

Universidade Federal Rural da Amazônia, Brasil

E-mail: raphael.lobato@outlook.com

Gustavo Schwartz

ORCID: https://orcid.org/0000-0002-1717-4491

Embrapa Amazônia Oriental, Brasil

E-mail: gustavo.schwartz@embrapa.br

\begin{abstract}
Resumo
Bancos de sementes do solo trazem informações sobre a flora atual e permitem cenários futuros sobre as comunidades vegetais. O objetivo deste trabalho foi determinar o percentual de germinação ex situ de um banco de sementes em diferentes níveis de luminosidade. A amostragem ocorreu em floresta primária explorada sob pousio no município de Moju, estado do Pará, Brasil. Foram coletadas 10 amostras compostas por quatro amostras simples em 10 parcelas da camada superficial do solo $(5 \mathrm{~cm})$. As amostras foram mantidas em casa de vegetação e distribuídas aleatoriamente em bandejas de isopor sob três condições de luminosidade: Sombra (casa de vegetação $+70 \%$ de sombrite); Sombra Média (casa de vegetação + 30\% de sobrite) e Pleno Sol, com cinco repetições cada. Após 120 dias, o banco de sementes apresentou 414 plântulas de 61 espécies, 48 gêneros e 34 famílias. Deste total, 27,78\% dos indivíduos germinaram nos três ambientes analisados. O ambiente com maior germinação de indivíduos foi Sombra Média $(\mathrm{F}=$ 140,$25 ; \mathrm{p}=0,0001 ; \mathrm{CV}=3,60 \%$ ), o qual também incluiu 48,15\% das espécies germinadas. $\mathrm{O}$ banco de sementes apresentou alta diversidade florística com predominância de espécies arbóreas, pioneiras e zoocóricas. Desta forma, condições ambientais determinam a germinação do banco de sementes, com destaque ao ambiente Sombra Média e às famílias Fabaceae, Rubiaceae e Urticaceae.
\end{abstract}

Palavras-chave: Bioma amazônico; Comunidade vegetal; Diversidade biológica; Florística.

\begin{abstract}
Soil seed banks bring information about the current flora and allow future scenarios about plant communities. The objective of this work was to determine the percentage of ex situ germination of a seed bank under different levels of light. Sampling took place in primary a harvested primary forest under fallow in the municipality of Moju, Pará state, Brazil. Ten samples composed of four simple samples were collected in 10 plots of the topsoil $(5 \mathrm{~cm})$. The samples were maintained in a greenhouse and randomly distributed in Styrofoam trays under three light conditions: Shade (greenhouse $+70 \%$ shade net); Medium Shade (greenhouse $+30 \%$ shade net), and Full Sun, with five repetitions each. After 120 days, the seed bank presented 414 seedlings of 61 species, 48 genera, and 34 families. From this total, $27.78 \%$ of the individuals germinated in the three environments analyzed. The environment with the highest germination of individuals was Medium Shade $(\mathrm{F}=140.25 ; \mathrm{p}=0.0001 ; \mathrm{CV}=3.60 \%)$, which also included $48.15 \%$ of the germinated species. The seed bank showed high floristic diversity with a predominance of tree, pioneer, and zoochoric species. In this way, environmental conditions determine the germination of the seed bank, with emphasis on the Medium Shade environment and the Fabaceae, Rubiaceae and Urticaceae families.
\end{abstract}

Keywords: Biome Amazonia; Plant community; Biological diversity; Floristics.

\section{Resumen}

Los bancos de semillas del suelo brindan información sobre la flora actual y permiten escenarios futuros sobre las comunidades de plantas. El objetivo de este trabajo fue determinar el porcentaje de germinación ex situ de un banco 
de semillas a diferentes niveles de luz. El muestreo se realizó en bosque primario explorado en barbecho en el municipio de Moju, estado de Pará, Brasil. Se recolectaron diez muestras compuestas por cuatro muestras simples en 10 parcelas de la capa superior del suelo $(5 \mathrm{~cm})$. Las muestras se mantuvieron en un invernadero y se distribuyeron aleatoriamente en bandejas de poliestireno bajo tres condiciones de luz: Sombra (invernadero + 70\% red de sombra); Sombra Media (invernadero + 30\% red de sombra) y Pleno Sol, con cinco repeticiones cada una. Después de 120 días, el banco de semillas presentó 414 plántulas de 61 especies, 48 géneros y 34 familias. De este total, 27,78\% de los individuos germinaron en los tres ambientes analizados. El ambiente con mayor germinación de individuos fue Sombra Media $(\mathrm{F}=140,25 ; \mathrm{p}=0,0001 ; \mathrm{CV}=3,60 \%)$, que también incluyó 48,15\% de las especies germinadas. El banco de semillas mostró una alta diversidad florística con predominio de especies arbóreas, pioneras y zoocóricas. De esta manera, las condiciones ambientales determinan la germinación del banco de semillas, con énfasis en el ambiente de Sombra Media y las familias Fabaceae, Rubiaceae y Urticaceae.

Palabras clave: Bioma amazónico; Comunidad vegetal; Diversidad biológica; Florística.

\section{Introdução}

O bioma amazônico apresenta um mosaico de vegetação de alta diversidade biológica, onde a cobertura vegetal é composta por um elevado número de espécies (Dionisio et al., 2016), muitas das quais endêmicas e praticamente ainda desconhecidas (Hubbell, 2013). No entanto, atividades antrópicas ocorridas nas últimas décadas têm provocado mudanças nas paisagens, com consequente perda de diversidade biológica. Para a manutenção do equilíbrio dos ecossistemas, se faz necessária a interação entre habitantes locais e seu ambiente, incluindo a variada composição florística e rica diversidade de espécies (Hubbell, 2013). Desse modo, o banco de sementes é um dos responsáveis pela estrutura e composição florística dos ambientes em regeneração natural, por garantir a diversidade das espécies, o estabelecimento dos grupos ecológicos e a restauração de sua riqueza.

O estoque de sementes viáveis no solo, em dada área e momento, é definido como banco de sementes, o qual varia de acordo com a dispersão e germinação (Diogo et al., 2015; Costa et al., 2020; Santos et al., 2017) e com o ambiente (Bertolini et al., 2020; Linê et al., 2020). O banco de sementes do solo é composto por serapilheira e sementes não germinadas, que podem estar em estado de dormência. Sua estrutura e composição de espécies são determinadas pelas características florísticas e pelos filtros ambientais, os quais determinam o recrutamento das espécies vegetais (Faist \& Collinge, 2015). Portanto, as sementes chegam ao ambiente por meio da chuva de sementes, dos agentes dispersores e pelos indivíduos vegetais pioneiros na exploração do ambiente, os quais na maioria das vezes não estão mais presentes na composição florística avançada na sucessão ecológica (Franco et al., 2012). Em uma dada área, a perpetuação e interação entre a dispersão de sementes e seu acúmulo no solo geram um banco de reserva da diversidade florística constituída por indivíduos de diferentes espécies e famílias.

A determinação da flora de um banco de sementes é importante para o conhecimento da vegetação autóctone, manutenção da diversidade genética nas comunidades e populações, e promoção da sucessão ecológica. O conhecimento da flora do banco de sementes pode auxiliar na regeneração de ecossistemas que sofreram algum tipo de antropização. Assim, informações obtidas com a germinação do banco de sementes em ambientas artificiais podem contribuir para o conhecimento da dinâmica de florestas. Nesse sentido, o objetivo deste trabalho foi determinar a taxa de germinação ex situ e a flora do banco de sementes do solo de uma floresta em diferentes níveis de luminosidade.

\section{Metodologia}

\section{Área de Estudo}

A coleta de dados foi realizada em ambiente de floresta primária na tipologia Floresta Ombrófila Densa, na Fazenda Agroecológica São Roque (coordenadas $3^{\circ} 03^{\prime}$ 14,85" S e 48 59’ 36,15" O), município de Moju, microrregião de Tomé-Açú, estado do Pará, Brasil. A área de 1.433,33 ha é formada por 1.016,70 ha de reserva legal, 339,63 ha de uso alternativo e 77,00 ha de áreas de proteção permanente. A reserva legal no período de 2007 a 2010 passou por intervenção silvicultural a partir de 
um Plano de Manejo Florestal Sustentável (PMFS) em Unidade de Produção Anual (UPA) única composta de 22 unidades de trabalho (UT) e, a partir de 2011, entrou em pousio. O clima da região é do tipo Ami (quente úmido), segundo a classificação de Köppen, com temperatura média anual de $25^{\circ} \mathrm{C}$ a $27^{\circ} \mathrm{C}$ e precipitação média anual de $2.000 \mathrm{~mm}$ a $3.000 \mathrm{~mm}$ (Andrade et al., 2017).

\section{Demarcação das Áreas de Coleta}

Com base em informações do inventario florestal pré-exploratório, mapas de uso do solo e microzoneamento, foram selecionadas quatro unidades de trabalho para a coleta de dados. Para garantir a qualidade e representatividade do banco de sementes, foram utilizadas unidades amostrais de forma circular. A partir do ponto de referencia (indivíduos de Copaifera reticulata) foi estabelecida uma circunferência de $17,5 \mathrm{~m}$ de raio (área $=962,11 \mathrm{~m}^{2}$ ) com pontos marcados nas direções Norte, Sul, Leste e Oeste. A área total amostrada foi de 9.621,15 $\mathrm{m}^{2}$ em 10 árvores. Em seguida, no interior de cada quadrante foi marcado aleatoriamente um ponto de coleta do banco de sementes, levando sempre em consideração a distancia mínima de $1 \mathrm{~m}$ do tronco da árvore referencia.

\section{Amostragem do banco de sementes do solo}

Em 10 árvores foram coletadas quatro amostras (uma amostra por quadrante/árvore) de serapilheira, juntamente com a camada superficial do solo até a profundidade de $5 \mathrm{~cm}$. A coleta foi realizada em janeiro de 2014 com o auxílio de um quadrado de PVC $(50 \mathrm{~cm}$ x $50 \mathrm{~cm}$ x $5 \mathrm{~cm})$ e uma pá de jardinagem. As 40 amostras simples de cada área de coleta foram homogenizadas em 10 amostras compostas, acondicionadas em sacos plásticos e levadas para a casa de vegetação do Horto de Plantas Medicinais da Embrapa Amazonia Oriental, Belém, PA. Posteriormente foram distribuidas aleatoriamente em bandejas de isopor ( $36 \mathrm{~cm}$ x $25 \mathrm{~cm}$ x $5 \mathrm{~cm}$ ) furadas contendo uma camada de terra preta, dispostas em bancadas a $1 \mathrm{~m}$ de altura do solo e submetidas a três diferentes condições de luminosidade: i) Sombra $-\mathrm{S}$ (casa de vegetação $+70 \%$ de sombrite; ii) Sombra Média SM (casa de vegetação $+30 \%$ de sobrite e iii) Pleno Sol - PS. Cada tratamento foi composto por cinco repetições com cinco bandejas cada, perfazendo 75 unidades amostrais. Foi realizada irrigação diária, ou de acordo com a necessidade e, quando observado a falta de germinação, o material era revolvido. A contagem do número de plantas germinadas foi realizada quando plantas apresentaram estruturas morfológicas que facilitassem o reconhecimento da espécie. Após a determinação botânica, as plântulas emergidas foram retiradas das bandejas para não interferirem na germinação de novos indivíduos. As espécies não identificadas foram transplantadas para sacos separadamente para posterior identificação taxonômica.

\section{Análise de dados}

A avaliação da germinação foi realizada semanalmente por um período de quatro meses por meio do percentual do número total de plântulas emergentes. Para a normalização, os valores de porcentagem de germinação foram transformados em $\sqrt{\mathrm{Y}+0,5}$ e submetidos à análise de variância (ANOVA), com aplicação do teste de Tukey, em nível de 5\% de significância. A amostragem da composição florística incluiu as espécies germinadas e identificadas em cada nível de sombreamento. As espécies foram identificadas por um parataxonomista e com o auxílio de literatura específica, corrigidos e atualizados através do banco de dados do Missouri Botanical Garden (www.mobot.org/tropicos) e Flora Do Brasil (2020) de acordo com APG IV. A caracterização da síndrome de dispersão das espécies seguiu os critérios propostos por Pijl (1982), sendo empregadas as seguintes categorias: autocoria (Auto), anemocoria (Anemo) e zoocoria (Zoo). Com base na literatura também foram verificados o hábito e categorias sucessionais das plantas.

Foram determinadas as seguintes variáveis fitossociológicas: densidade, riqueza (Menhinick; Margalef), Dominância (Berger-Parker) e heterogeneidade floristica do banco de sementes (Shannon-Wiener - H'). O índice de Shannon-Wiener foi 
calculado com base no número de indivíduos de cada espécie e no total dos indivíduos amostrados. Também foram calculados seguintes índices: a) Equidade de Pielou, b) Alfa de Fisher, que varia de 0 a 1, indicando o valor máximo de diversidade de espécies em relação ao número de indivíduos amostrados e c) índice de Brillouin. Os índices obtidos para cada ambiente (S, SM e PS) foram posteriormente comparados em duplas utilizando os índices de diversidade de Shannon-Wiener de duas amostras, por meio de teste t descrito por Hutcheson (1970), Poole (1974), Magurran (1988).

\section{Resultados e Discussão}

\section{Composição florística do Banco de sementes}

Foram encontrados 414 indivíduos germinados em todas as 75 unidades de amostras/bandejas em casa de vegetação, as quais representam 61 espécies pertencentes a 49 gêneros e 34 famílias botânicas. Fabaceae foi representada por seis espécies, seguida por Cyperaceae, Euphorbiaceae, Rubiaceae e Solanaceae (4), Melastomataceae e Passifloraceae (3) e Annonaceae, Piperaceae, Salicaceae, Sapindaceae e Urticaceae (2). As famílias Araceae, Asteraceae, Bignoniaceae, Cannabaceae, Chrysobalanaceae, Commelinaceae, Crassulaceae, Dioscoreaceae, Hypericaceae, Lacistemataceae, Lauraceae, Linderniaceae, Loganiaceae, Malpighiaceae, Marantaceae, Menispermaceae, Moraceae, Myristicaceae, Phyllanthaceae, Plantaginaceae, Primulaceae e Rhamnaceae foram representadas por uma espécie cada. Foi identificada uma espécie (conhecida vulgarmente como douradinha) em nível de família (Rubiaceae) e duas plantas não foram identificadas nem mesmo em nível de família (Tabela 1).

Tabela 1. Famílias e espécies vegetais presentes no banco de sementes do solo e germinadas em casa de vegetação. Hábito (Hab): Arbórea (Arb), Herbácea (Herb) e Arbusto (Abt). Sindrome de dispersão (SD): Zoocoria (Zoo), Anemocoria (Ane) e Autocoria (Aut). Gupo Ecológico (GE): Pioneira (P), Secundária (S), Secundária inicial (Si), Secundária tardia (St), Clímax (Cl) e Tolerante a sombra (Ts). Ambientes de Germinação (AG): Sombra (S), Sombra Média (SM) e Pleno Sol (PS) e Número de indivíduos $(\mathrm{Ni})$.

\begin{tabular}{|c|c|c|c|c|c|c|c|}
\hline \multirow[t]{2}{*}{ Familia } & \multirow[t]{2}{*}{ Espécie } & \multirow[t]{2}{*}{ Hab } & \multirow[t]{2}{*}{ SD } & \multirow[t]{2}{*}{ GE } & \multicolumn{3}{|c|}{ AG/Ni } \\
\hline & & & & & $\mathbf{S}$ & SM & PS \\
\hline \multirow[t]{2}{*}{ Annonaceae } & Xylopia frutescens Aubl. & Arb & Zoo & - & 1 & 0 & 0 \\
\hline & Xylopia nítida Dunal & Arb & Zoo & $\mathrm{P}$ & 0 & 10 & 0 \\
\hline Araceae & Philodendron sp. & Her & Zoo & $\mathrm{Si}$ & 0 & 1 & 0 \\
\hline \multirow[t]{2}{*}{ Asteraceae } & Bidens cynapiifolia & Her & Ane & - & 0 & 0 & 1 \\
\hline & Kunth & & & & & & \\
\hline \multirow[t]{2}{*}{ Bignoniaceae } & Jacaranda copaia & Arb & Zoo & $\mathrm{P}$ & 1 & 2 & 0 \\
\hline & (Aubl.) D.Don & & & & & & \\
\hline \multirow[t]{2}{*}{ Cannabaceae } & Trema micrantha (L.) & Arb & Zoo & $\mathrm{P}$ & 2 & 16 & 0 \\
\hline & Blum & & & & & & \\
\hline Chrysobalanaceae & Licania sp. & Arb & Zoo & S & 11 & 0 & 0 \\
\hline Commelinaceae & Commelina sp. & Her & Zoo & Ts & 1 & 0 & 1 \\
\hline \multirow[t]{2}{*}{ Crassulaceae } & Bryophyllum pinnatum & Her & - & Ts & 0 & 3 & 0 \\
\hline & (Lam.) Oken & & & & & & \\
\hline \multirow[t]{2}{*}{ Cyperaceae } & Cyperus laxus Lam. & Her & - & - & 0 & 0 & 2 \\
\hline & Cyperus luzulae (L.) & Her & Ane & $\mathrm{P}$ & 0 & 0 & 1 \\
\hline
\end{tabular}


Rottb. Ex Retz.

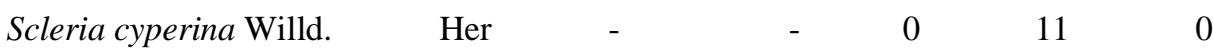

ex Kunth

$\begin{array}{lllllll}\text { Scleria pterota var. } & \text { Her } & \text { Ane } & \text { Ts } & 4 & 1 & 1\end{array}$

melaleuca (Rchb. Ex

Schltdl.\& Cham.)

Uittien

\begin{tabular}{|c|c|c|c|c|c|c|c|}
\hline Dioscoreaceae & Dioscorea sp. & Arb & - & $\mathrm{Ni}$ & 0 & 3 & 0 \\
\hline \multirow[t]{5}{*}{ Euphorbiaceae } & Croton obtusus Rusby & Arb & - & $\mathrm{Si}$ & 0 & 3 & 0 \\
\hline & Croton sp. & Arb & Zoo & $\mathrm{P}$ & 1 & 0 & 14 \\
\hline & Croton trinitatis Millsp. & Arb & Aut & $\mathrm{P}$ & 7 & 0 & 0 \\
\hline & Euphorbia prostrata & Her & Zoo & $\mathrm{P}$ & 0 & 0 & 24 \\
\hline & Aiton & & & & & & \\
\hline \multirow[t]{6}{*}{ Fabaceae } & Dinizia excelsa Ducke & Arb & Zoo & $P$ & 0 & 2 & 0 \\
\hline & Inga graciliflora Benth. & Arb & Zoo & $\mathrm{Si}$ & 1 & 0 & 0 \\
\hline & $\begin{array}{l}\text { Desmodium barbatum } \\
\text { (L.) Benth. }\end{array}$ & Her & Zoo & $\mathrm{P}$ & 1 & 2 & 2 \\
\hline & $\begin{array}{l}\text { Desmodium guianense } \\
\text { (Aubl.) DC. }\end{array}$ & Arb & Aut & $\mathrm{P}$ & 0 & 0 & 2 \\
\hline & $\begin{array}{l}\text { Stylosanthes guianensis } \\
\text { (Aubl.) Sw. }\end{array}$ & Her & Aut & $\mathrm{P}$ & 0 & 0 & 8 \\
\hline & Mimosa sensitiva $\mathrm{L}$. & Her & - & $\mathrm{P}$ & 0 & 3 & 0 \\
\hline Hypericaceae & $\begin{array}{l}\text { Vismia guianensis } \\
\text { (Aubl.) Pers. }\end{array}$ & Arb & Zoo & $\mathrm{P}$ & 7 & 17 & 2 \\
\hline Lacistemataceae & $\begin{array}{l}\text { Lacistema pubescens } \\
\text { Mart }\end{array}$ & Arb & Zoo & $\mathrm{Ni}$ & 5 & 3 & 0 \\
\hline Lauraceae & Ocotea $\mathrm{sp}$. & Arb & Zoo & $\mathrm{Ni}$ & 5 & 7 & 0 \\
\hline Linderniaceae & $\begin{array}{l}\text { Lindernia crustacea (L.) } \\
\text { F. Muell. }\end{array}$ & Her & - & $\mathrm{P}$ & 7 & 3 & 2 \\
\hline Loganiaceae & Spigelia anthelmia $\mathrm{L}$. & Her & - & St & 0 & 0 & 10 \\
\hline Malpighiaceae & $\begin{array}{l}\text { Stigmaphyllon } \\
\text { martianum A. Juss. }\end{array}$ & Her & - & - & 0 & 0 & 8 \\
\hline Marantaceae & $\begin{array}{l}\text { Ischnosiphon gracilis } \\
\text { (Rudge) Körn }\end{array}$ & Abt & Zoo & $\mathrm{CL}$ & 2 & 0 & 0 \\
\hline \multirow[t]{4}{*}{ Melastomataceae } & $\begin{array}{l}\text { Clidemia hirta (L.) D. } \\
\text { Don }\end{array}$ & Abt & Zoo & $\mathrm{P}$ & 1 & 3 & 0 \\
\hline & Miconia ciliata (Rich.) & Abt & Zoo & Ts & 0 & 2 & 0 \\
\hline & $\mathrm{DC}$ & & & & & & \\
\hline & $\begin{array}{l}\text { Miconia silicicola } \\
\text { Gleason }\end{array}$ & Abt & Zoo & $\mathrm{P}$ & 1 & 0 & 0 \\
\hline
\end{tabular}




\begin{tabular}{|c|c|c|c|c|c|c|c|}
\hline Menispermaceae & $\begin{array}{l}\text { Cissampelos } \\
\text { andromorpha DC. }\end{array}$ & Abt & Zoo & $\mathrm{P}$ & 0 & 2 & 0 \\
\hline Moraceae & $\begin{array}{l}\text { Clarisia ilicifolia } \\
\text { (Spreng.) Lanj. \& } \\
\text { Rossberg }\end{array}$ & Arb & - & - & 1 & 0 & 0 \\
\hline Myristicaceae & Virola michelii Heckel & Arb & - & $\mathrm{St}$ & 0 & 2 & 0 \\
\hline \multirow[t]{3}{*}{ Passifloraceae } & $\begin{array}{l}\text { Passiflora glandulosa } \\
\text { Cav. }\end{array}$ & Arb & Zoo & - & 0 & 3 & 0 \\
\hline & Passiflora sp. & Arb & Zoo & $\mathrm{P}$ & 0 & 3 & 0 \\
\hline & $\begin{array}{l}\text { Piriqueta cistoides (L.) } \\
\text { Griseb }\end{array}$ & Her & - & - & 0 & 0 & 7 \\
\hline Phyllanthaceae & Phyllanthus niruri L. & Art & Aut & $\mathrm{P}$ & 2 & 11 & 13 \\
\hline \multirow[t]{2}{*}{ Piperaceae } & $\begin{array}{l}\text { Peperomia pellucida } \\
\text { (L.) Kunth }\end{array}$ & Her & Zoo & $\mathrm{Si}$ & 0 & 2 & 0 \\
\hline & Piper peltatum $\mathrm{L}$. & Arb & Zoo & $S$ & 0 & 1 & 0 \\
\hline Plantaginaceae & Scoparia dulcis $\mathrm{L}$. & Her & - & $\mathrm{Si}$ & 10 & 0 & 2 \\
\hline Primulaceae & $\begin{array}{l}\text { Clavija ruiziana } \\
\text { (Kuntze) Mez }\end{array}$ & Arb & - & - & 0 & 1 & 0 \\
\hline Rhamnaceae & $\begin{array}{l}\text { Gouania cornifolia } \\
\text { Reissek }\end{array}$ & Arb & - & $\mathrm{P}$ & 0 & 3 & 0 \\
\hline \multirow[t]{5}{*}{ Rubiaceae } & $\begin{array}{l}\text { Borreria latifolia } \\
\text { (Aubl.) K. Schum }\end{array}$ & Her & Aut & $\mathrm{P}$ & 0 & 0 & 4 \\
\hline & $\begin{array}{l}\text { Borreria verticillata }(\mathrm{L} .) \\
\text { G. Mey. }\end{array}$ & Her & Aut & $\mathrm{P}$ & 0 & 0 & 1 \\
\hline & $\begin{array}{l}\text { Douradinho (nome } \\
\text { vulgar) }\end{array}$ & - & - & - & 0 & 5 & 0 \\
\hline & $\begin{array}{l}\text { Oldenlandia lancifolia } \\
\text { (Schumach.) DC. }\end{array}$ & Her & - & $\mathrm{P}$ & 1 & 2 & 2 \\
\hline & $\begin{array}{l}\text { Palicourea marcgravii } \\
\text { A. St.-Hil. }\end{array}$ & Arb & Zoo & $\mathrm{Si}$ & 0 & 2 & 0 \\
\hline Salicaceae & $\begin{array}{l}\text { Banara guianensis Aubl. } \\
\text { Homalium guianense } \\
\text { (Aubl.) Oken }\end{array}$ & $\begin{array}{l}\text { Arb } \\
\text { Arb }\end{array}$ & $\begin{array}{l}\text { Zoo } \\
\text { Zoo }\end{array}$ & $\mathrm{P}$ & $\begin{array}{l}0 \\
10\end{array}$ & 4 & 0 \\
\hline Sapindaceae & $\begin{array}{l}\text { Cupania sp. } \\
\text { Serjania paucidentata } \\
\text { DC. }\end{array}$ & $\begin{array}{l}\text { Arb } \\
\text { Arb }\end{array}$ & $\begin{array}{l}\text { Zoo } \\
-\end{array}$ & $\begin{array}{l}\mathrm{Si} \\
\mathrm{P}\end{array}$ & $\begin{array}{l}4 \\
5\end{array}$ & $\begin{array}{l}0 \\
1\end{array}$ & $\begin{array}{l}0 \\
0\end{array}$ \\
\hline \multirow[t]{4}{*}{ Solanaceae } & Solanum caavurana Vell & Art & Zoo & $\mathrm{P}$ & 1 & 1 & 0 \\
\hline & Solanum juripeba Rich. & Art & Zoo & $\mathrm{P}$ & 1 & 5 & 0 \\
\hline & Solanum sp. & Arb & Zoo & $\mathrm{P}$ & 6 & 0 & 0 \\
\hline & Solanum subinerme Jacq & Arb & Zoo & $\mathrm{P}$ & 0 & 6 & 0 \\
\hline
\end{tabular}




\begin{tabular}{|c|c|c|c|c|c|c|c|}
\hline \multirow[t]{5}{*}{ Urticaceae } & Bryophyllum calcynum & Her & - & - & 0 & 1 & 0 \\
\hline & Cecropia obtusa Trécul & Arb & Zoo & $\mathrm{P}$ & 1 & 33 & 1 \\
\hline & Cecropia palmata Willd. & Arb & Zoo & $\mathrm{P}$ & 0 & 3 & 0 \\
\hline & Cecropia peltata $\mathrm{L}$. & Arb & Zoo & $\mathrm{P}$ & 0 & 3 & 0 \\
\hline & $\begin{array}{l}\text { Pourouma } \\
\text { guianensisAubl. }\end{array}$ & Arb & Zoo & $\mathrm{P}$ & 4 & 2 & 0 \\
\hline NI & $\mathrm{NI}$ & - & - & - & 0 & 9 & 0 \\
\hline Total & & & & & 104 & 202 & 108 \\
\hline
\end{tabular}

Fonte: Autores.

O maior número de indivíduos foi apresentado por Cecropia obtusa (35), Phyllanthus niruri (26), Vismia guianensis (26), Euphorbia prostrata (24), Trema micrantha (18), Croton sp. (15), Homalium guianense (14), Lindernia crustacea (12), Ocotea sp. (12), Scoparia dulcis (12), Licania sp. (11), Scleria cyperina (11), Xylopia nítida (10) e Spigelia anthelmia (10). Para Fonseca et al. (2006), conhecer as condições de luminosidade que promovem a maior taxa de germinação das sementes e melhor desenvolvimento das plântulas de cada espécie é essencial para o sucesso de atividades de reflorestamento e de plantio em florestas naturais.

Dos 414 individuos germinados nos três ambientes, foram identificados 229 indivíduos com hábito arbóreo, 168 herbáceo e 17 arbustivo. Braga et al. (2011) também observou maior número de indivíduos arbóreos do que herbáceos em estudo de serapilheira para recuperação de áreas degradadas. Quanto aos níveis de luminosidade, do total dos indivíduos germinados nos três ambientes (414), a maioria 137 (59,83\%) dos exemplares arbóreos germinou em Sombra Média (SM). Por outro lado, plantas de hábito herbáceo predominaram no ambiente a Pleno Sol (PS) (31,87\%) e as arbustivas se fizeram presentes apenas a Sombra (S) (Tabela 3). Para Paz et al. (2016), o predomínio de espécies arbóreas e herbáceas presentes no solo das florestas tropicais pode ser uma importante fonte de recrutamento de plântulas após alguma perturbação e auxiliar na regeneração das florestas.

Para a síndrome de dispersão, no total dos três ambientes, foram observadas 226 individuos zoocóricos, 46 autocóricos, 12 anemocóricos e 130 não foram determinados. No ambiente PS o predomínio foi de espécies autocóricas (56,52\%), em SM zoocóricas (55,75\%) e em S as anemocóricas (41,67\%). Para essa característica, 47,69\% das espécies não foram identificadas e a maioria estava presente em SM. Esses resultados mostram que as características morfológicas e ecológicas das espécies contribuem para a ocorrência dos difererntes tipos de dispersão. Quanto ao Grupo Ecológico, houve predominância das espécies pioneiras, com 268 individuos. Desse total 52,99\% germinaram em SM, seguido de 27,61\% em PS (Tabela 2). 
Tabela 2. Percentagem de indivíduos por Ambiente de Germinacao (AG) quanto ao Hábito (Hab.), Sindrome de dispersão (SD) e Grupo Ecológico (GE). AG: Sombra (S), Sombra Média (SM) e Pleno Sol (PS). Hab.: Arbusto (Abt), Arbóreo (Arb) e Herbáceo (Herb). SD: Anemocoria (Ane), Autocoria (Aut), Zoocoria (Zoo) e Não idedificado (Ni). GE: Pioneira (P), Secundária (S), Secundária inicial (Si), Secundária tardia (St), Clímax (Cl), Tolerante a sombra (Ts) e Clímx (Cl).

\begin{tabular}{llllllllllllll}
\hline & \multicolumn{3}{c}{ Hab } & \multicolumn{1}{c}{ SD } & \multicolumn{1}{c}{ GE } \\
\hline AG & Abt & Arb & Herb & Ane & Aut & Zoo & Ni & P & S & Si & St & Ts & Cl \\
\hline S & 0,00 & 1,87 & 7,26 & 1,67 & 9,57 & 4,34 & 6,92 & 9,40 & 5,00 & 0,00 & 0,00 & 4,48 & 0,00 \\
SM & 0,00 & 59,83 & 29,76 & 25,00 & 23,91 & 55,75 & 47,69 & 52,99 & 25,00 & 32,00 & 0,00 & 24,14 & 50,00 \\
OS & 0.00 & 8,30 & 2,00 & 33,33 & 56,52 & 19,91 & 25,38 & 27,61 & 0,00 & 8,00 & 0,00 & 41,38 & 50,00 \\
\hline
\end{tabular}

Fonte: Autores.

\section{Germinação do banco de sementes}

Ao longo do tempo de observação, verificou-se que os ambientes estudados (S, SM e PS) influenciaram significativamente na germinação do banco de sementes em nível de família ( $F=8,25 ; p=0,0056$; CV = 11,86\%), espécie ( $F$ $=22,88 ; \mathrm{p}=0,0001 ; \mathrm{CV}=7,62 \%)$ e indivíduo $(\mathrm{F}=140,25 ; \mathrm{p}=0,0001 ; \mathrm{CV}=3,60 \%)$, pelo teste Tukey, com predominância do ambiente SM.

Considerando todos os ambientes, das 61 espécies identificadas, $48,15 \%$ ocorreram em SM, superior a S $(27,16 \%)$ e PS (24,69\%). Nessa mesma condição germinou o maior número de indivíduos (202), representando 48,79\% do total de 414, sendo superior aos ambientes S e PS (Figura 1). Esses resultados mostram que as condições ambientais exercem influência na taxa de germinação das espécies do banco de sementes do solo em casa de vegetação. As condições controladas, como os de telas sombreadoras, são úteis para analisar a tolerância das diferentes espécies ao sol ou sombra. Porém, experimentos nesses microambientes nem sempre irão refletir integralmente as condições do ambiente natural, tanto pelas diferenças na luz disponível quanto em outros aspectos edáficos e climáticos.

Figura 1. Percentual de plantas emergidas do banco de sementes nos ambientes: Sombra, Sombra Média e Pleno Sol em casa de vegetação. Letras indicam diferenças nas médias de famílias, espécies e indivíduos entre diferentes ambientes.

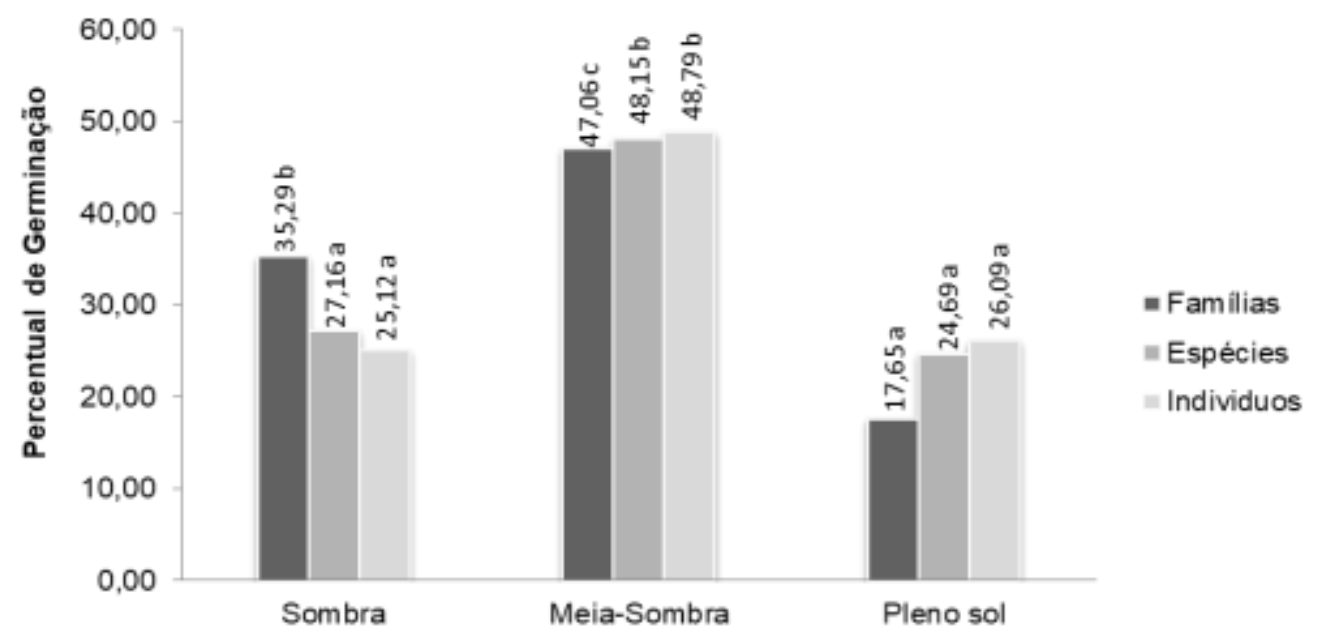

Niveis de luminosidade

Fonte: Autores. 
Ao se observar o percentual de germinação, uma mesma espécie germinou em mais de um ambiente. Scleria pterota var. melaleuca (Cyperaceae), Desmodium barbatum (Fabaceae), V. guianensis (Hyperianceae), L. crustacea (Linderniaceae), P. niruri (Phyllanthaceae), Oldenlandia lancifolia (Rubiaceae) e C. obtusa (Urticaceae) germinaram independentemente das condições do ambiente (S, SM e PS). De outro modo, espécies de Bignoniaceae, Commelinaceae, Euphorbiaceae, Lacistemataceae, Lauraceae, Melastomataceae, Plantaginaceae, Salicaceae, Sapindaceae e Solanaceae emergiram sob dois ambientes e, as demais famílias apresentaram espécies apenas em um dos ambientes (Tabela 1). Espécies que germinaram em três ambientes apresentaram plasticidade, sendo esta uma qualidade de grande importância ecológica. Para Rossatto e Kolb (2010), espécies vegetais com ampla plasticidade em caracteres ligados a fatores de sobrevivência apresentam grandes vantagens adaptativas em ambientes de transição, heterogêneos e instáveis.

O desdobramento mostrou que $35,29 \%, 26,23 \%$ e $23,19 \%$ de famílias, espécies e indivíduos, ocorreram em dois ambientes, respectivamente. Em três ambientes verificou-se a presença de 23,53\% das famílias, 11,48\% das espécies e 27,78\% de indivíduos e os demais foram exclusivas de uma única condição dos ambientes de estudo (Tabela 3).

Tabela 3. Percentual do número de famílias, espécies e indivíduos germinados em casa de vegetação nos ambientes. Sombra (S), Sombra Média (SM) e Pleno Sol (PS).

\begin{tabular}{llllllll}
\hline & \multicolumn{7}{c}{ Ambiente de Germinação (\%) } \\
\cline { 2 - 8 } & $\mathbf{S}$ & SM & PS & S+SM+PS & S+SM & S+PS & SM+PS \\
\hline Família & 8,82 & 23,53 & 8,82 & 23,53 & 26,47 & 5,88 & 2,94 \\
Espécies & 14,75 & 31,15 & 16,39 & 11,48 & 16,39 & 4,92 & 4,92 \\
Indivíduos & 8,21 & 22,22 & 16,43 & 27,78 & 19,08 & 4,11 & 0,00 \\
\hline
\end{tabular}

Fonte: Autores.

\section{Diversidade florística e Ambiente}

Em SM a flora do banco de sementes foi representada por 26 famílias, 33 gêneros e 42 espécies. No ambiente S o banco de sementes apresentou 22 famílias, 26 gêneros e 29 espécies e no ambiente PS a flora foi representada por 14 famílias, 18 gêneros e 21 espécies. Quanto à diversidade para os ambientes S, SM e PS em relação ao número de espécies entre tratamentos, o fator ambiente foi determinante na germinação do maior número de espécies, favorecido principalmente em SM. Por outro lado, S e PS apresentaram quantidades semelhantes de espécies e individuos, indicando que estes dois índices analisados independem do ambiente, o qual para a região se apresenta como um fato positivo. Os índices apresentados auxiliaram na caracterização da comunidade vegetal observada, mostrando que o banco de sementes nos três ambientes de estudo apresenta heterogeneidade florística, alta diversidade e elevada dominância ecológica (Tabela 4). 
Tabela 4. Índices fotissociológicos do banco de sementes floresta primária explorada sob pousio em Moju, PA, Brasil e germinados em três ambientes ex situ em diferentes níveis de luminosidade. Sombra (S), Sombra Média (SM) e Pleno Sol (PS).

\begin{tabular}{llll}
\hline & \multicolumn{3}{c}{ Ambiente } \\
\cline { 2 - 4 } & $\mathbf{S}$ & SM & PS \\
\hline Espécies & 29 & 42 & 108 \\
Indivíduos & 104 & 202 & 0,1087 \\
Dominância (D) & 0,06028 & 0,05852 & 0,8913 \\
Simpson (1-D) & 0,9397 & 0,9415 & 2,536 \\
Shannon (H') & 3,02 & 3,274 & 0,6017 \\
Equidade H' & 0,7063 & 0,6289 & 2,273 \\
Brillouin & 2,658 & 2,973 & 2,021 \\
Menhinick & 2,844 & 2,955 & 4,272 \\
Margalef & 6,029 & 7,724 & 0,8331 \\
Equidade J & 0,8967 & 0,8759 & 7,776 \\
Fisher Alpha & 13,34 & 16,12 & 0,2222 \\
Berger-Parker & 0,1058 & 0,1634 & 22,88 \\
Chao-1 & 48,5 & 43,91 & \\
\hline
\end{tabular}

Fonte: Autores.

Na comparação entre pares de ambientes, os valores do índice de Shannon-Wiener indicaram alta significância para os pares SM x PS e S x PS e significância para S x SM mostrando existir boa diversidade nesses ambientes para o número de espécies e indivíduos. Porém pelo índice de Simpson apenas o SM x PS foi significante (Tabela 5).

Tabela 5. Teste de diversidade entre pares dos níveis de luminosidade pelos índices de Shannon-Wiener e Simpson, pelo teste t. Sombra (S), Sombra Média (SM) e Pleno Sol (PS).

\begin{tabular}{|c|c|c|c|c|c|c|c|}
\hline \multicolumn{2}{|c|}{ Índices } & \multicolumn{2}{|c|}{$\begin{array}{l}\text { Pares dos tratamentos } \\
\text { S x SM }\end{array}$} & \multicolumn{2}{|l|}{$S \times O S$} & \multicolumn{2}{|l|}{ SM $\times \mathbf{O S}$} \\
\hline \multirow{5}{*}{ 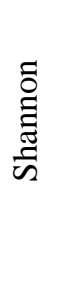 } & $\mathrm{H}^{\prime}$ & 3,0196 & 3,2739 & 3,0196 & 2,5365 & 3,2739 & 2,5365 \\
\hline & Var & 0,0066853 & 0,005288 & 0,0066853 & 0,008688 & 0,005875 & 0,0088688 \\
\hline & $\mathrm{T}$ & $-2,3244$ & & 3,8965 & & 6,2382 & \\
\hline & gl & 252,31 & & 209,40 & & 233,27 & \\
\hline & $\mathrm{p}$ & $0,020899 *$ & & $0,00013131 * *$ & & $2,06 \mathrm{E}-09 * * *$ & \\
\hline \multirow{5}{*}{ 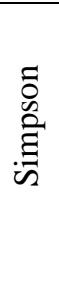 } & $\mathrm{D}$ & 0,060281 & 0,058524 & 0,060281 & 0,10871 & 0,058524 & 0,10871 \\
\hline & Var & $4,76 \mathrm{E}-05$ & $5,65 \mathrm{E}-05$ & 4,76E-05 & 0,000202 & $5,65 \mathrm{E}-05$ & 0,000202 \\
\hline & $\mathrm{T}$ & 0,17224 & & $-3,0636$ & & $-3,1193$ & \\
\hline & $\mathrm{gl}$ & 288,39 & & 155,81 & & 169,71 & \\
\hline & $\mathrm{p}$ & 0,86337 & & 0,0025775 & & $0,0021306^{* *}$ & \\
\hline
\end{tabular}

$* \mathrm{p}<0,05 ; * * \mathrm{p}<0,01 \mathrm{e} * * * \mathrm{p}<0,001$. Fonte: Autores. 


\section{Conclusão}

Os resultados obtidos sugerem que as condições ambientais ex situ determinam o percentual de germinação do banco de sementes do solo, com predominancia do ambiente Sombra Média para as famílias, espécies e indivíduos. Contudo, Scleria pterota var. melaleuca (Cyperaceae), Desmodium barbatum (Fabaceae), Vismia guianensis (Hyperianceae), Lindernia crustacea (Linderniaceae), Phyllanthus niruri (Phyllanthaceae), Oldenlandia lancifolia (Rubiaceae) e Cecropia obtusa (Urticaceae) germinam independente das condições do ambiente (Sombra, Sombra Média e Pleno Sol), variando apenas quanto ao número de indivíduos. A composição florística do banco de sementes apresenta alta diversidade com predominância de espécies arbóreas, do grupo ecológico das pioneiras com dispersão zoocorica.

\section{Agradecimentos}

Agradecemos à estudante de doutorado Ana Cristina Magalhães de Carvalho (in memorian) por sua essencial colaboração neste trabalho.

\section{Referências}

Andrade, V. M. S., Cordeiro, I. M. C. C., Schwartz, G., Rangel-Vasconcelos, L. G. T., \& Oliveira, F. de A. (2017). Considerações sobre o clima e aspectos edafoclimáticos da mesorregião nordeste paraense. In: Cordeiro, I. M. C. C., Rangel-Vasconcelos, L. G. T., Schwartz, G., Oliveira, F. de A. (eds.) Nordeste Paraense: Panorama Geral e Uso Sustentável das Florestas Secundárias. Belém: EDUFRA, p.59-86.

Bertolini, I. C., Watzlawick, L. F., Sens, T. M. Z. G., Vantroba, A. P., Schran, J. A., Pott, C. A., \& Lustosa, S.B.C. (2020). Composição florística do banco de sementes em área de recuperação aos dez anos de idade no sul do país. Research, Society and Development, 9(5), e91953174 http://dx.doi.org/10.33448/rsdv9i5.3174

Braga, A. J. T., Griffith, J. J., Paiva, H. N., Silva, F. C., Corte, V. B., \& Meira Neto, J. A. A. (2011). Enriquecimento do sistema solo-serapilheira com espécies arbóreas aptas para recuperação de áreas degradadas. Revista Árvore, 31(6), 1145-1154. https://doi.org/10.1590/S0100-67622007000600019

Costa, P., Pereira, Z., Santos, B., Fernandes, S., Fróes, C., \& Barbosa, T. (2020). Banco de sementes do solo em áreas restauradas no sul do estado de Mato Grosso do Sul - MS. Ciência Florestal, 30(1), 104-106. http://dx.doi.org/10.5902/1980509832896

Diogo, I. J. S., Fortunato, E. M. M., \& Costa, I. R. (2015). Seed deposition in the edge interior gradientof a degraded fragment of tropical semideciduous forest, Northeastern Brazil. Revista de Biología Tropical, 63(4), 981-994.

Dionisio, L., Filho O., Crivelli, B., Gomes, J., Oliveira, M., \& Carvalho, J. (2016). Importância fitossociológica de um fragmento de floresta ombrófila densa no estado de Roraima, Brasil. Agro@mbiente, 10(3), 243-252. http://dx.doi.org/10.18227/1982-8470ragro.v10i3.3381

Faist, A. M., \& Collinge, S.K. (2015). Seed bank composition varies along invasion and inundation gradients in vernal pool wetlands. Plant Ecology, 216 (4), 553-564.

Flora do Brasil. Flora do Brasil 2020 em construção. Jardim Botânico do Rio de Janeiro. http://floradobrasil.jbrj.gov.br/

Fonseca, M. G, Leão, N. V. M, \& Santos, F. A. M. (2006). Germinação de sementes e crescimento inicial de plântulas de Pseudopiptadenia psilostachya (DC.) G.P. Lewis \& M.P. Lima (Leguminosae) em diferentes ambientes de luz. Revista Árvore, 30(6), 885-891.

Franco, B. K. S., Martins, S. V., Faria, P. C. L., \& Ribeiro, G. A. (2012). Densidade e composição florística do banco de sementes de um trecho de floresta estacional semidecidualno campus da Universidade Federal de Viçosa, Viçosa, MG. Revista Árvore, 36(3), 423-432. https://doi.org/10.1590/S010067622012000300004

Hubbell, S. P. (2013). Tropical rain forest conservation and the twin challenges of diversity and rarity. Ecology and evolution, 3(10), 3263-3274. https://doi.org/10.1002/ece3.705

Hutcheson, K. (1970). A test for comparing diversities based on the Shannon formula. Journal of Theoretical Biology, 29, $151-154$.

Linê, J. D. B., Fernandes, S. S. L., Mello, L. S., Zolin, T. D. S., \& Pereira, Z. V. (2020). Banco de sementes do solo em três fitofisionomias de cerrado no leste de Mato Grasso do Sul. Research, Society and Development, 9(9), e546997291 http://dx.doi.org/10.33448/rsd-v9i9.7291

Magurran, A. 1988. Ecological diversity and its measurement. Princeton: Princeton University Press.

Paz, G. V., Silva, K. A., \& Almeida-Cortez, J. S. de. (2016). Banco de sementes em áreas de caatinga com diferentes graus de antropização no setão de Itaparica-Pe. Journal of Environmmental Analysis and Progress, 1(1), 61-69. https://doi.org/10.24221/jeap.1.1.2016.987.61-69

Pijl, L. V. D. (1982). Principles of dispersal in higher plants. (3a ed.) Springer Verlag.

Poole, R.W. (1974). An introduction to quantitative ecology. McGraw-Hill. 
Research, Society and Development, v. 10, n. 1, e22710111523, 2021

(CC BY 4.0) | ISSN 2525-3409 | DOI: http://dx.doi.org/10.33448/rsd-v10i1.11523

Rossatto, D. R., \& Kolb, R. M. (2010). Gochnatiapolymorpha (Less.) Cabrera (Asteraceae) cambia en la estructura de la hoja debido a las diferencias en las condiciones de luz y edáficas. Acta Botanica Brasilica, 24(3), 605-612. https://doi.org/10.1590/S0102-33062010000300002

Santos, G. L., Pereira, M. G., Conto, T. de, Carvalho, D. C. de, Amorim, T. A. de, Mendonça, V. M. M., \& Moraes, L. F. D. de. (2017). Influência da pedoforma na composição do banco de sementes em floresta secundária na região de Mar de Morros, Pinheiral - RJ. Ciência Florestal, 27(4), 1217-1228. http://dx.doi.org/10.5902/1980509830310 